\title{
Cyatbidium meteorensis spec. nov., ein neuer Crinoie aus der Familie Holopodidae
}

\author{
H. FECHTER \\ Abteilung für Wirbellose Tiere, Zoologische Staatssammlung München; \\ München, Bundesrepublik Deutschland
}

\begin{abstract}
Cyatbidium meteorensis spec. nov, a new crinoid of the family Holopodidae. The new species, dredged on the eastward slope of the Great Meteor Bank, must be referred to the genus Cyathidium SteENstrup, 1847, which seemed to have become extinct at the end of the mesozoic era. The rigid theca is without any sutures; it varies in shape according to the substrate to which it adheres, from low broad-based, narrowing upwards or cylindrical, to conical with tapering base. The crinoid has a smooth surface, an oral field with orals and 10 arms. Primibrachials $(\mathrm{IBr})$ are singular, axillary, low, trapezium shaped and very stout. First secundibrachials $\left(\mathrm{IIBr}_{1}\right)$ are triangular and vary in size; they are smaller and not as stout as the IBr. But like these, they have a series of arched grooves or ridges at the lateral surfaces. The second secundibrachials are considerably smaller than the first ones and articulated orad perpendicular to them. $\mathrm{IBr}$ and $\mathrm{IrBr}_{1}$ can form a solid cover across the oral field, protecting and hiding the spirally coiled smaller arms beneath. The pinnules are broadened, leaf-shaped, margins able to curl up. The outer sides of tentacles are supported by finger-like sclerites. The juveniles resemble closely those of Holopus rangi D'OrbigNY; they have two IBr, the second one divides and becomes IIBr.
\end{abstract}

\section{EINLEITUNG}

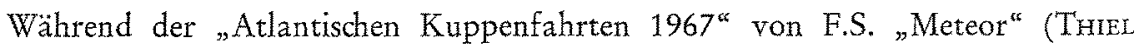
1970) wurden auf der Großen Meteor Bank mit einer Tonnendredge auf den Stationen Nr. 173 and 178 insgesamt 20, teilweise beschädigte Exemplare einer seßhaften, bisher unbekannten Crinoiden-Art (Abb. 1) heraufgebracht, der insofern größere Bedeutung zukommt, als Arten jener Gattung, der diese neue Art zugeordnet werden muß, bisher nur fossil - aus der Kreidezeit - bekannt sind und es sich daher um ein sog. „lebendes Fossil" handelt.

\section{FUNDORT}

Die beiden Fundorte liegen auf der Ostflanke der Großen Meteor Bank und haben folgende Koordinaten: Station $\mathrm{Nr}, 173$ : $29^{\circ} 42,2^{\prime} \mathrm{N}$; $28^{\circ} 20,5^{\prime} \mathrm{W}$; Fangtiefe $850-580 \mathrm{~m}$. Station Nr. 178: $29^{\circ} 57,9^{\prime} \mathrm{N} ; 2^{\circ} 15,7^{\prime} \mathrm{W}$; Fangtiefe $690-610 \mathrm{~m}$. Der Untergrund bestand aus hartem Felsgestein, allem Anschein nach Basalt. 


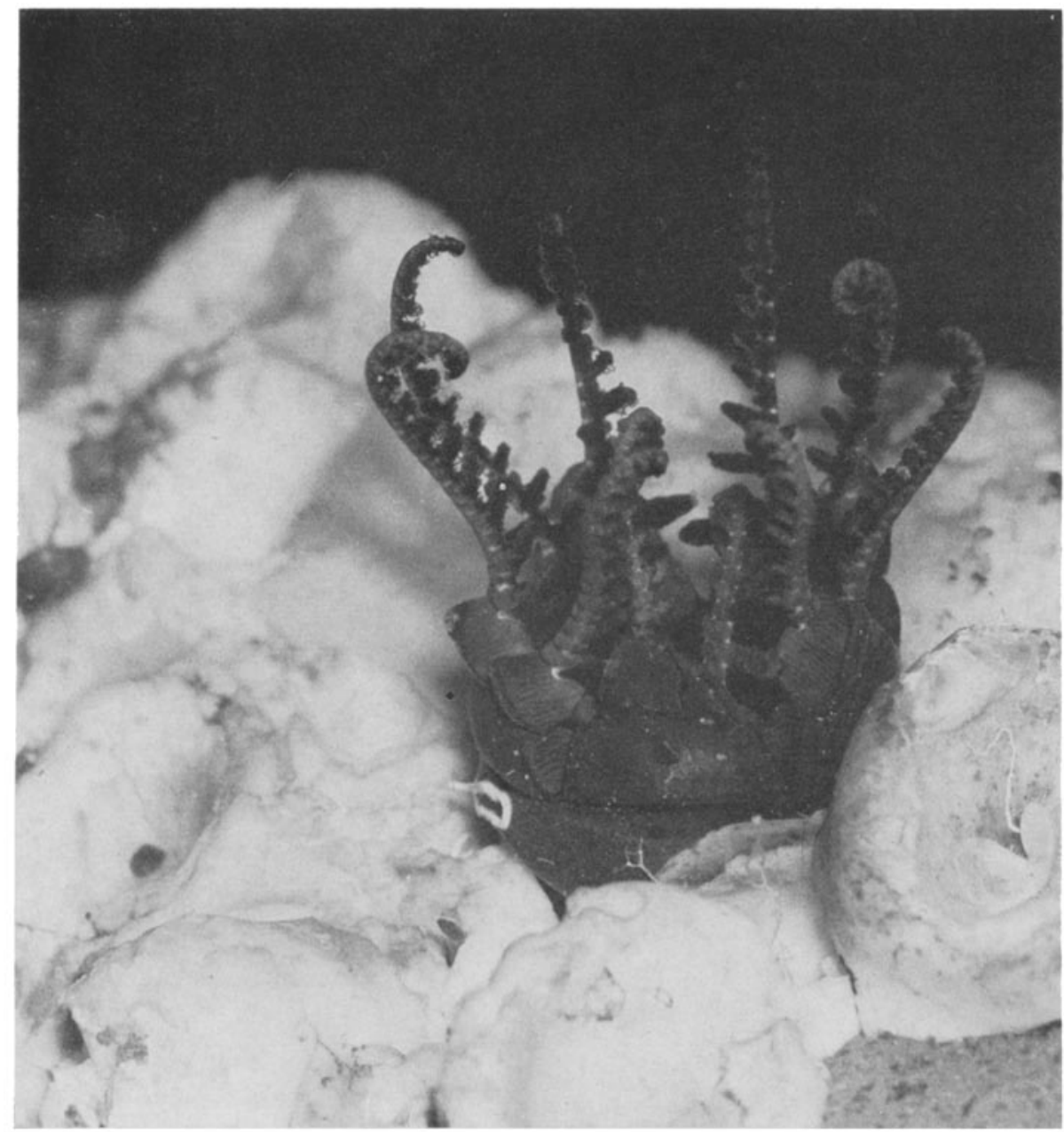

Abb. 1: Cyathidium meteorensis. Nach dem Fang lebend photographiert, mit nahezu voll entfalteten Armen, umgeben von leeren, ausgebleichten Theken abgestorbener Artgenossen; am Grund der Theka links unten im Bild ein juveniles Tier. (Aufnahme Dr. M. Graßhoff, Frankfurt/M.)

\section{BESCHREIBUNG}

Theka

Die becherförmige, vermutlich aus den nahtlos verwachsenen Radialia gebildete, dickwandige Theka sitzt bei manchen Individuen mit verbreiterter Basis direkt auf hartem Fels, wobei die aufragende Theka zylindrisch oder nach oben schwach konisch 


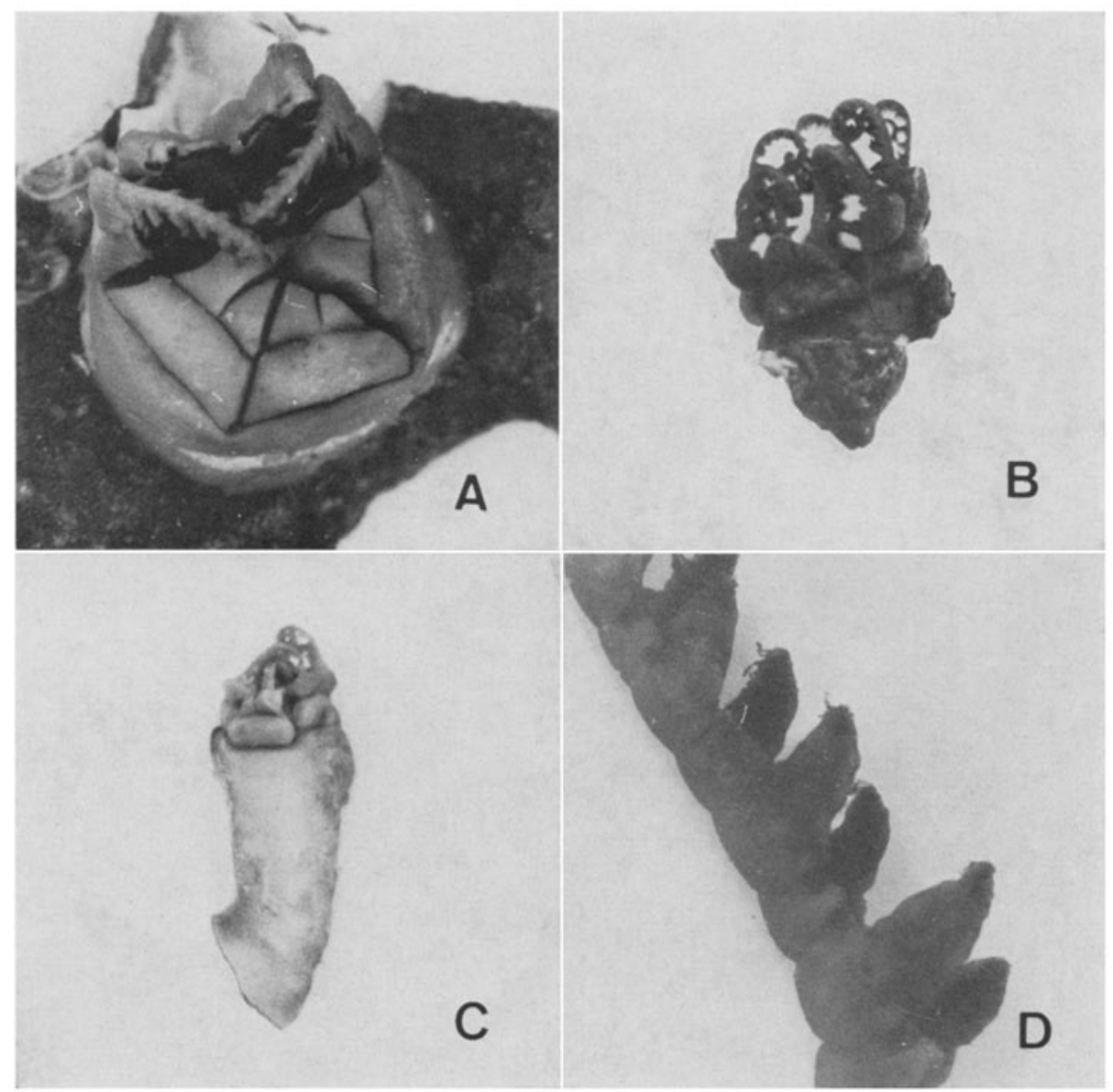

Abb. 2: Cyathidium meteorensis. Die je nach Substrat verschiedenen Thekaformen und Teil eines Armes mit Pinnulae. A breitbasig, direkt auf Fels. B orad konisch erweitert, in Theken abgestorbener Artgenossen. C kelchförmig, in Spalten. D Armstïck mit Pinnulae

zulaufend gestaltet sein kann, während bei anderen, die sich in den ausgebleichten Theken abgestorbener Artgenossen angesiedelt haben, sich die basale Theka aborad mehr oder weniger stark konisch verschmälert. Ein Exemplar, das in einer Felsspalte heranwuchs, weist ebenfalls eine aborad konisch zulaufende Theka auf. Die Gestalt der Theka wird demzufolge in gewissem Ausmaß von der Form des Substrates mitbestimmt (Abb. 2, Tab. 1). Die Theka wird bei der Mehrzahl der Individuen rundherum nicht gleichmäßig hoch. Vielmehr ist eine Thekenseite auf eine Bogenlänge von etwa zwei bis drei Radien und den eingeschlossenen Interradien höher als die restliche, gegenüberliegende Thekenwand - eine Asymmetrie, die sich bei den Armen wiederholt. Der Theka-Querschnitt ist kreisförmig rund, die äußere Begrenzungslinie der Basis, den Substratverhältnissen entsprechend, unregelmäßig. Die gesamte Oberfläche 
der Theka ist völlig glatt, nicht skulpturiert. Am schmalen Thekarand sind zum ThekaInneren hin, schräg abfallend, annähernd trapezförmige Gelenkflächen für die Primibrachialia (IBr) eingekerbt. Durch die fünf ebenen, adorad abgeschrägten Gelenkflädhen erhält der obere Theka-Innenrand eine pentagonale Form. Interradial, jeweils zwischen den benachbarten Gelenkflächen, erhebt sich ein kleiner, keilförmiger, dreieckiger Vorsprung, dessen Spitze in interradialer Richtung nach außen weist, den Thekaoberrand jedoch nicht überragt. Die Dicke der Thekenwand nimmt bei den Formen mit verbreiterter Basis in aboraler Richtung beträchtlich zu, wogegen die aborale Basis selbst äußerst dünn bleibt, so daß der Leibesinhalt hier häufig nur durch eine hauchdünne, wenig oder gar keine Skelettsubstanz enthaltende Körperwand vom Untergrund getrennt ist.

Tabelle 1

Dimensionen (mm) der Theka für die drei in Abbildung 2 gezeigten Formen von Cyathidium meteorensis

\begin{tabular}{lccc|}
\hline Exemplar & $\mathrm{a}$ & $\mathrm{b}$ & $\mathrm{c}$ \\
\hline Durchmesser an der Basis & 14 & 0 & 9 \\
Durchmesser am Oberrand & 11,5 & 16,7 & 10 \\
Höhe der Theka & 6 & 15 & 18 \\
\hline
\end{tabular}

\section{Primibrachialia}

Pro Radius ist jeweils nur ein Primibrachiale (IBr) ausgebildet. Es ist niedrig, breit-trapezförmig, sehr dick und auf der glatten Außenseite aborad gewölbt. Die leicht konkave Oralseite trägt die beiden vom distalen Rand adorad schräg abfallenden Gelenkflächen für die ersten Sekundibrachialia. Auf der Oralseite der IBr gabelt sich die Ambulacralfurche. Im Winkel der Gabelung befindet sich eine pyramidenartige Erhebung. Die interradiaden, einander zugewandten Seitenflächen sind mit bogig verlaufenden Furchen und Leisten versehen.

\section{Sekundibrachialia}

Die ersten Sekundibrachialia $\left(11 B r_{1}\right)$ eines $I B r$ sind infolge ungleichmäßiger Teilung fast immer verschieden groß und auch im Umriß nicht ganz identisch. Das eine ist proximal meist breiter als das andere und radiad auch länger. Sie haben annähernd die Gestalt eines niedrigen, dreieckigen Prismas; die aborale Oberfläche ist glatt. Die interradiaden und die einander zugewandten Innenseiten weisen die gleiche Riefung auf wie die IBr. Das zweite Sekundibrachiale ( $\mathrm{IIBr}_{2}$ ) ist gegenüber dem $\mathrm{IIBr}_{1}$ abrupt stark verschmälert; von $\mathrm{da} a \mathrm{~b}$ werden die weiteren IIBr distad nur allmählich schmächtiger. Im Querschnitt sind sie halbmondförmig. An einem Arm wurden bis zu 39 Bradhiale gezählt. In der Armlänge besteht wie bei der Thekenhöhe, eine nicht immer gleich deutlich ausgeprägte Asymmetrie. Das IIBr 2 inseriert am $\mathrm{IIBr}_{1}$ orad nahezu senkrecht. 
In Ruhestellung bedecken die zehn $\mathrm{IIBr}_{1}$, zusammen mit den fünf $\mathrm{IBr}$, die Mundfläche vollständig, während sich die restlichen Armabschnitte in der Oral-Aboral-Ebene von den distalen Enden her spiralig unter die von den $\mathrm{IBr}+\mathrm{IIBr}_{1}$ gebildeten Deckstücke einrollen.

Im Gegensatz zu den meisten anderen Crinoiden besitzt das $\operatorname{IIBr}_{1}$ auf der Außenseite (interradiad) eine Pinnula, die meist sogar die mächtigste überhaupt ist.

\section{Pinnulae}

Die Pinnulae sind stark blattartig verbreitert und inserieren mit breiter Basis am Brachiale, wobei die Pinnula-Achse fast senkrecht zum Brachiale steht, oder nur schwach zentrifugad geneigt ist. Die mundnahen Pinnulae sind mächtiger als die mundferneren. Am größten ist $P_{1}$, am zweitgrößten $P_{2}$, wie überhaupt die Pinnulae der Außenseiten durchwegs geringfügig größer erscheinen als die der Innenseiten. Bei einem Exemplar ist $P_{1}$ allerdings auffallend klein; die Ursache dafür konnte ich noch nicht ermitteln, hoffe sie jedoch durch histologische Untersuchungen klären zu können.

Die Pinnulae werden durch breite, schuppenförmig flache Pinnulare gestïtzt. In den mundnahen Pinnulae sind es meist 3--2, selten 4 Stïck, in den mundferneren nimmt die Zahl über 2 bis auf $1 \mathrm{ab}$. Die inneren Pinnulae haben in der Regel weniger Pinnulare als die äußeren. An die schuppenförmigen Pinnulare schließt sich distal ein annähernd dreieckiger, lappiger Abschnitt an, der mehrere kleine Sklerite enthält.

Wie bei allen Crinoiden haben die Ambulacralfüßchen auf den Pinnulae die Funktion von Tentakeln. Sie sind nicht in Dreiergruppen (Triaden) angeordnet. Außergewöhnliche Größenunterschiede, wie sie in den Triaden anderer Crinoiden vorkommen, sind ebenfalls nicht vorhanden. Es wurden bis zu 26 Tentakel auf einer Pinnula gezählt. Die Tentakel sind proximal stämmig, distal peitschenförmig dünn ausgezogen, mit Papillen besetzt und fast alle dick in einen Schleimmantel gehïllt, der mit Detritus durchsetzt ist. Auf ihrer Außenseite werden sie von fingerförmigen, vom Rande der Pinnulare ausgehenden Skleriten gestützt. Der Pinnularand kann zur Pinnula-Achse hin eingerollt werden, wobei sich die fingerförmigen Sklerite auf den Außenseiten schützend über die eingekrümmten und stark kontrahierten Tentakel legen. Am mundnahen (zentripetalen) Pinnularand stehen mehr Tentakel als am mundfernen (zentrifugalen) und zwar deshalb, weil sie am zentripetalen Rand die Pinnulafurche in einem Bogen bis zur Armfurche begleiten und hier bis zu 4 Tentakel auf dem Brachiale stehen. Am Rand der Armfurche desselben Brachiale erhebt sich gegenüber der Einmündung der Pinnulafurche eine Gruppe aus 2-m, meist 3 kurzen Tentakeln, die offensichtlich das Überschießen und Herausfallen der von der Pinnula herantransportierten Nahrungspartikel verhindern sollen.

\section{Mundfeld und Ambulacralrinne}

Das Mundfeld ist in aboraler Richtung stark schüsselförmig eingesenkt. Neigen sich nun die $\mathrm{IBr}+\mathrm{IIBr}_{1}$ über dem Mundfeld zusammen und bilden auf diese Weise einen Deckel, so entsteht $z$ wischen diesem. Deckel und dem Mundfeld eine große, gerun- 
det napfförmige bis konische Höhle (Mundfeldhöhle), in der bequem die in der OralAboral-Ebene eingerollten Arme untergebracht werden können. Die interradialen Sektoren enden orad mit Klappen, die deltaförmige, den Oralia wahrscheinlich homologe Sklerite enthalten. Die Mundöffnung ist verhältnismäßig weit; der After, klein und unauffällig, mündet nicht, wie bei den Crinoiden häufig, auf einem Analkonus, sondern öffnet sich trichterförmig in die Mundfeldebene.

Die Ambulacralrinne ist im Mundfeldbereich tief und schmal, auf den Armen dagegen sehr seicht und relativ breit. Entlang ihrer Ränder sind keine Sacculi zu erkennen.

Die thekale Leibeshöhle selbst, d. h. der Raum unter dem Mundfeld, zwischen diesem und der Körperinnenwand, ist sehr eng und bietet den dort liegenden Organen nur wenig Platz. Ober Bau und Anordnung des thekalen Leibesinhaltes wird in einer weiteren Untersuchung berichtet.

\section{Juvenile Tiere}

Unter dem Material befinden sich auch einige juvenile Individuen. Sie sind niedrig, schildförmig, mit glatter Oberfläche. Auffallenderweise besitzen sie 2 Primibrachialia, von denen das $\mathrm{IBr}_{2}$ axillar ist und einige wenige IIBr trägt, die äußerst kurze Ärmchen bilden. Die weitere Entwicklung geht dahin, daß sich die $\mathrm{IBr}_{2}$ perradial spalten und auf diese Weise bei den Adulten zu $\mathrm{IIBr}_{1}$ werden. Aus dieser nachträglichen Teilung der $\mathrm{IBr}_{2}$ resultieren vermutlich auch die Ungleichmäßigkeiten der späteren $\mathrm{IBBr}_{1}$.

\section{Färbung}

Die Farbe der frisch gefangenen Tiere ist schwarz, mit grünlich-violett changierendem Schimmer. In den Konservierungsflüssigkeiten (Formol, Äthanol) wird der Farbstoff sehr stark extrahiert; es entsteht eine tief purpurviolette Lösung. Die Tiere enthalten im Vergleich zu anderen Crinoiden sehr große Farbstoffmengen. Vollständig ausgelaugte Teile sind schmutzig-weiß.

\section{Stellung der Arme beim Nahrungsfang}

In der inaktiven Phase sind die distalen Armteile eingekrümmt unter dem schützenden Schild aus $\mathrm{IBr}+\mathrm{IIBr}_{1}$ verborgen. Einige der gefangenen Individuen entfalteten sich jedoch nach einiger Zeit (Abb. 1), so daß die Haltung der Arme, wie sie wohl auch beim Nahrungsfang eingenommen wird, beobachtet werden konnte. Die entrollten Arme sind dabei mehr oder weniger steil aufgerichtet, divergieren etwas nach oben und bewegen sich nicht.

Hierbei zeigt sich auch, daß die Arme eines Triviums etwas länger als die des restlichen Biviums sind. Eine Asymmetrie, die auch bei den übrigen Holopodidae auftritt.

Die breiten Pinnulae sind seitlich weit abgespreizt, die Tentakel lang ausgestreckt und schleimbedeckt. 
$\mathrm{Da}$ die $I I B r_{2}$ an den $I I B r_{1}$ orad fast senkrecht $\mathrm{zu}$ deren Längsachse ansetzen, müssen die $I_{1 B r} r_{1}$ desto weiter nach außen gekippt werden, je stärker sich der aus den Armen gebildete Reusentrichter noch oben öffnet. Dem Offnungswinkel sind sehr enge mechanische und geometrische Grenzen gesetzt.

\section{SYSTEMATISCHE ZUORDNUNG UND VERWANDTSCHAFTS- BEZIEHUNGEN}

Die aus den Radialia nahtlos fest zusammengefügte, ohne Stiel direkt am Untergrund festgewachsene, massige, cirrenlose Theka und die stets nur in der Einzahl und axillar ausgebildeten Primibrachialia, die 10 Arme sowie das Fehlen synarthrialer bzw. syzygialer Verbindungen zwischen den Brachialia, kennzeichnen die Art als zur Familie Holopodidae gehörig.

Die Ubereinstimmung in den Besonderheiten der Armstruktur - wie den dreieckigen, im Vergleich zu den übrigen IIBr großen $\mathrm{IIBr}_{1}$, die zusammen mit den IBr im oralwärts geklappten Zustand über dem Mundfeld und den eingerollten, distalen Armabschnitten einen schützenden Deckel bilden - mit den Verhältnissen bei der bisher nur fossil, aus der Kreidezeit (Cenoman, Turon, Santon, Maastricht, Dan) bekannten Gattung Cyathidium STEenstrup 1847 ist so vollständig, daß man die neue Art zwangsläufig dieser "alten" Gattung zuordnen muß. Cyatbidium meteorensis ist somit z. Z. als einzige überlebende Art einer Gattung zu betrachten - aus der Kreidezeit wurden 3 Arten beschrieben (RASMUSSEN 1961) - die am Obergang zum Tertiär bereits ausgestorben schien.

In der Familie Holopodidae gab es bisher nur eine einzige rezente Art: Holopus rangi D'ORBIGNY, die in westindischen Gewässern vorkommt. Trotz des auf den ersten Blick weitgehend verschiedenen Aussehens der adulten Tiere von $H$. rangi und $C$. meteorensis weisen die Einzelheiten des Konstruktionsprinzips auf eine sehr nahe Verwandtschaft der beiden Gattungen hin. Wie eng die Verwandtschaftsbeziehungen zu $H$. rangi sind, wird vor allem dadurch unterstrichen, daß die juvenilen Tiere in ihrer äußeren Morphologie - abgesehen von der Granulation der Oberfläche bei den jungen H. rangi, die den C. meteorensis fehlt - weitgehend übereinstimmen. Beide sind niedrig, schildförmig und die $\mathrm{IBr}_{1}$ noch nicht axillar.

Die Ergebnisse einer eingehenden Untersuchung der neuen Art werden in der Reihe D der "Meteor"-Forschungsergebnisse veröffentlicht.

Als Holotypus wurde das in Abbildung 2B gezeigte Exemplar von der Station Nr. 178 gewählt. Es wird in der Abteilung für wirbellose Tiere der Zoologischen Staatssammlung München unter der Sachkatalog-Nummer: Echinodermata 585/1 aufbewahrt.

\section{ZUSAMMENFASSUNG}

1. Die neue Crinoidenart aus der Familie Holopodidae (Fundort: Ostflanke der GroBen Meteor Bank) ist der Gattung Cyatbidium Stennstrup 1847 zuzuordnen, die seit der Kreidezeit als ausgestorben galt. 
2. Die Gestalt der nahtlos fest zusammengefügten Theka ist je nach Substrat niedrig, breitbasig, nach oben verschmälert bzw. zylindrisch oder oralwärts konisch erweitert, kelchförmig. Die Oberfläche ist glatt, und das Mundfeld mit Oralia versehen.

3. Die Art besitzt 10 Arme. Die axillaren, niedrig-trapezförmigen, sehr massigen Primibrachialia (IBr) sind in der Einzahl. Die ersten Sekundibrachialia ( $\left.\mathrm{IIBr}_{1}\right)$ sind dreieckig, meist ungleich groß, ebenfalls dick und wie die $\mathrm{IBr}$ an den einander zugewandten Seiten bogig gerieft. Die zweiten Sekundibrachialia haben eine abrupt schmälere Form als die ersten und stehen orad senkrecht zu ihnen. $\mathrm{IBr}$ und $\mathrm{IIBr}_{1}$ sind in der inaktiven Phase über das Mundfeld geklappt und bilden einen schützenden Dedkel, unter dem die schmäleren Armabschnitte spiralig eingerollt verborgen sind.

4. Die Pinnulae mit ihren einrollbaren Rändern verbreitern sich blattartig. Die Tentakeln sind gleichgestaltig und werden auf der Außenseite durch fingerförmige Sklerite gestïtzt.

5. Die juvenilen Tiere gleichen im wesentlichen denen von Holopus rangi D'ORBIGNY. Sie besitzen zwei IBr; aus dem zweiten gehen durch Spaltung die späteren $\mathrm{IIBr}_{1}$ hervor.

Danksagungen. Herr Dr. M. Grasshoff, Frankfurt/M., hat mir freundlicherweise einige photographische Aufnahmen zur Verfügung gestellt; Herr Dr. L. TIefenbacher, München, war mir bei Photoarbeiten behilflich. Ich danke beiden Herren herzlich.

\section{ZITIERTE LITERATUR}

RASmussen, H. W., 1961. A monograph on the cretaceous Crinoidea. Biol. Skr. 12, 238-248. THIEL, Hj., 1970. Bericht über die Benthosuntersuchungen während der „Atlantischen Kuppenfahrten $1967^{“ 6}$ von F.S. „Meteor ${ }^{4}$. Meteor ForschErgebn. (D) 7, 23-42.

Anschrift des Autors: Dr. H. FrCHTER

Zoologische Staatssammlung München

8 München 19

Maria-Ward-Straße 1b

Bundesrepublik Deutschland 